\title{
Behavior of Top-Blown Jet under Reduced Pressure
}

\author{
Ikuhiro SUMI, ${ }^{1)}$ Goro OKUYAMA, ${ }^{2)}$ Seiji NABESHIMA, ${ }^{1)}$ Hidetoshi MATSUNO ${ }^{2)}$ and Yasuo KISHIMOTO ${ }^{1)}$ \\ 1) Steelmaking Research Dept., Steel Research Laboratory, JFE Steel Corporation, 1 Kokan-cho, Fukuyama, Hiroshima 721 - \\ 8510 Japan. $\quad$ 2) 1 Kawasaki-cho, Chuo-ku, Chiba, Chiba 260-0835 Japan.
}

(Received on July 24, 2006; accepted on September 20, 2006)

\begin{abstract}
In steelmaking, the top-blown oxygen jet is used in many different refining processes and its behavior is therefore an important factor in steel refining. Numerous studies have been conducted on jet behavior under atmospheric pressure conditions; however, its behavior under reduced pressure for vacuum refining processes is still not fully understood.

In this study, jet behavior under reduced pressure was investigated by measuring the dynamic pressure of the jet. Also, numerical simulation, which was used for jet simulation in recent studies, was carried out to clarify the details of the jet behavior. The simulated results were compared with the measured data and the numerical simulation method was validated.

As a result, the jet behavior was modeled using the numerical simulation, and the detailed effects of ambient pressure on the jet behavior were clarified. The potential core of the jet lengthened as the ambient pressure decreased, indicating that jet attenuation under reduced pressure is smaller than that under atmospheric pressure. Using this model, the effect of the nozzle shape on the post combustion behavior of the actual $\mathrm{RH}$ top-blown process could be explained.
\end{abstract}

KEY WORDS: steelmaking; RH; VOD; supersonic jet; model; vacuum; reduced pressure; measurement; lance; nozzle; velocity; Mach number; expansion; oxygen; numerical simulation; numerical experiment.

\section{Introduction}

In steelmaking, the top-blown oxygen jet is used in many different refining processes and its behavior is therefore an important factor in steel refining. The jet is normally a supersonic jet with a Laval nozzle used under atmospheric pressure. Jet behavior under atmospheric pressure conditions has been investigated for decades. ${ }^{1-5)}$ For example, an estimation model of jet behavior was built by Imai et al. for a small-scale experiment ${ }^{1)}$ and can be used to easily obtain jet velocity under atmospheric pressure.

In recent studies, numerical simulation of supersonic jets was possible due to rapid developments in numerical simulation technology. ${ }^{6-8)}$ In the BOF process, a multi-hole lance is normally used and the jet behavior is quite complex because of the suction effect. However, such complex behavior, including mutual interference of multiple jets, can be simulated using the numerical simulation method.

On the other hand, the top-blown oxygen jet is also applied to such vacuum refining processes as RH and VOD. When the ambient pressure of a vacuum refining process is reduced, the ambient density is also changed. Density is an important factor in jet behavior and the change in ambient density is considered to affect the jet behavior under reduced pressure. In addition, the difference between the ambient pressure and backpressure of the nozzle increases as the ambient pressure decreases. This causes an increase in the Mach number at the nozzle exit and affects the jet expansion behavior. Several studies have been conducted on jet behavior under reduced pressure; however, a quantitative understanding has not yet been attained..$^{9,10)}$

In this study, jet behavior under reduced pressure was investigated by measuring the dynamic pressure of the jet in a vacuum chamber as Exp. 1. In addition, numerical simulation was carried out to clarify the details of the jet. The simulated results were compared with the measured data in Exp. 1 and the numerical simulation method was validated.

Numerical simulation is a convenient method for obtaining details of the jet; however, a huge amount of time was required to obtain only a basic outline of the jet behavior. Moreover, a simpler model is useful for actual optimization of jet conditions. For that purpose, a numerical experiment was carried out. The jet behavior was modeled and the effects of ambient pressure on the jet behavior were clarified using the results obtained by numerical simulation. For validation of the jet model, a measurement experiment, Exp. 2, was carried out using a different nozzle from the one used in Exp. 1. Measured Mach number was in good agreement with the data calculated using the jet model.

Finally, the experiment was carried out using an actual $\mathrm{RH}$ operation, and post-combustion behavior could be explained using the jet model calculation.

\section{Experiment and Simulation Method}

\subsection{Measurement Experiment}

Figure 1 shows the experimental apparatus used to measure the dynamic pressure of the top-blown jet under reduced pressure. The lance used for the experiment was a one-hole Laval nozzle that was inserted into the chamber 


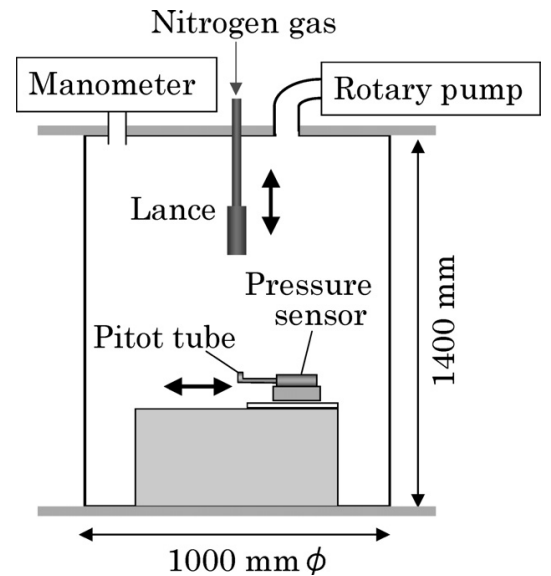

Fig. 1. Schematic view of experimental apparatus.

Table 1. Experimental conditions.

\begin{tabular}{|cc|c|c|c|c|c|}
\hline & & Exp. 1 & \multicolumn{4}{|c|}{ Exp.2 } \\
\hline \begin{tabular}{|} 
Ambient \\
pressure
\end{tabular}$\quad(\mathrm{kPa})$ & $\begin{array}{c}4,8,13, \\
40,101\end{array}$ & \multicolumn{4}{|c|}{4} \\
\hline $\mathrm{N}_{2}$ flow rate & $\left(\mathrm{Nm}^{3} / \mathrm{min}\right)$ & 0.49 & \multicolumn{5}{|c|}{0.44} \\
\hline Throat dia. & $(\mathrm{mm})$ & 2.6 & 3.0 & 3.0 & 3.0 & 3.0 \\
\hline Exit dia. & $(\mathrm{mm})$ & 7.5 & 7.5 & 6.0 & 5.0 & 4.0 \\
\hline $\mathrm{A}_{\mathrm{e}} / \mathrm{A}_{\mathrm{t}}$ & $(-)$ & 8.3 & 6.3 & 4.0 & 2.8 & 1.8 \\
\hline $\mathrm{P}_{\mathrm{ep}}$ & $(\mathrm{kPa})$ & 8.4 & 8.8 & 17.6 & 31.7 & 68.5 \\
\hline
\end{tabular}

from the top. Ambient pressure in the chamber was controlled using a rotary pump, and nitrogen was blown from the nozzle. The measurement device, which was equipped with a Pitot tube (JIS B8330) and a pressure sensor, was set in the chamber in advance. Inner diameter and outer diameter of the Pitot tube were 1.5 and $3.0 \mathrm{~mm}$, respectively. The measurement position was set by moving the lance vertically to measure the dynamic pressure distribution in the axial direction of the jet and the measurement device was scanned horizontally to measure the horizontal distribution of the dynamic pressure of the jet. Then, the distribution of Mach number, $M$, was calculated from the measured dynamic pressure, $P$, as follows ${ }^{11)}$ :

$$
M^{2}=\frac{2}{\gamma-1}\left[\left(\frac{P}{P_{\mathrm{e}}}\right)^{(\gamma-1) / \gamma}-1\right]
$$

where $P_{\mathrm{e}}$ is the ambient pressure and $\gamma$ is the ratio of heat capacity. For a supersonic jet, we made correction for the shockwave effect around the Pitot tube to calculate Mach number. ${ }^{12)}$

The experimental conditions are shown in Table 1. First, Exp. 1 was carried out. The nitrogen flow rate was set as constant and the ambient pressure was changed to clarify its effects on jet behavior. The optimum nozzle exit diameter for the correct expansion changes with the ambient pressure change because the Mach number at the nozzle exit changes as shown in Eq. (1). ${ }^{11)}$ In Exp. 1, the nozzle was designed for experimental conditions with $8 \mathrm{kPa}$ as the optimum ambient pressure. The results of Exp. 1 were used to validate the numerical simulation method used to build the jet model under reduced pressure.
Table 2. Conditions for numerical simulation.

\begin{tabular}{ll}
\hline Software, Model & $:$ Fluent 6, RSM, 2D-model \\
Medium & $:$ Nitrogen, 300K \\
Domain & $:$ 300mm $\phi \times 500 \mathrm{~mm}$ \\
Nozzle & $: 1$ hole, Laval nozzle \\
Nitrogen flow rate & $:$ 0.20, $0.35,0.49 \mathrm{Nm}^{3} / \mathrm{min}$ \\
Nozzle diameter & $:$ throat $2.6 \mathrm{~mm}$ \\
& exit $\quad 7.5 \mathrm{~mm}$ \\
Ambient pressure & $: 4,8,13,40,101 \mathrm{kPa}\left(0.49 \mathrm{Nm}^{3} / \mathrm{min}\right)$ \\
& $4,8,13,40 \mathrm{kPa}\left(0.35 \mathrm{Nm}^{3} / \mathrm{min}\right)$ \\
& $4,8,13 \mathrm{kPa}\left(0.20 \mathrm{Nm}^{3} / \mathrm{min}\right)$ \\
\hline
\end{tabular}

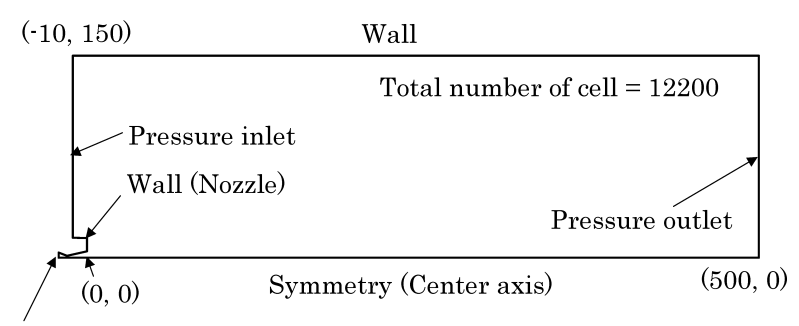

Pressure inlet (Gas inlet)

Fig. 2. Domain and boundary conditions.

Next, Exp. 2 was carried out. The nitrogen flow rate was set at $0.44 \mathrm{Nm}^{3} / \mathrm{min}$ and the ambient pressure at $4 \mathrm{kPa}$. Experimental apparatus and other basic conditions were the same as those of Exp. 1. In this experiment, we used several different nozzles that had the same throat diameter and a different exit diameter to investigate the effects of the nozzle exit diameter. The ambient pressure for correct expansion of each nozzle differs as shown in Table 1. Using these nozzles, the dynamic pressure was measured at $x=300 \mathrm{~mm}$, and the Mach number was calculated from the measured dynamic pressure. $x$ is the lance height that means the distance from the nozzle exit in the axial direction. The results were used to validate the jet model built in this study.

\subsection{Numerical Simulation}

Numerical simulation was also carried out for the same conditions as in Exp. 1; the simulation conditions are shown in Table 2. FLUENT 6 software was used as the CFD code with an unmodified Reynolds Stress Model, RSM. Domain and boundary conditions are shown in Fig. 2. The results of simulation using a gas flow rate of $0.49 \mathrm{Nm}^{3} / \mathrm{min}$ were compared with the measured data obtained in Exp. 1 to validate the numerical simulation method. Numerical simulation was also carried out using gas flow rates of 0.20 and $0.35 \mathrm{Nm}^{3} / \mathrm{min}$ to investigate the effects of gas flow rate on jet behavior. These simulated results under the reduced pressure were used for modeling the jet behavior under reduced pressure.

\section{Results and Discussion}

\subsection{Measured and Simulated Results}

Simulated distribution of Mach number on the center axis of the jet is shown in Fig. 3 with the Mach number measured in Exp. 1. The simulated results are in good agreement with the measured data. It was clarified that the potential core length increases and jet attenuation is sup- 


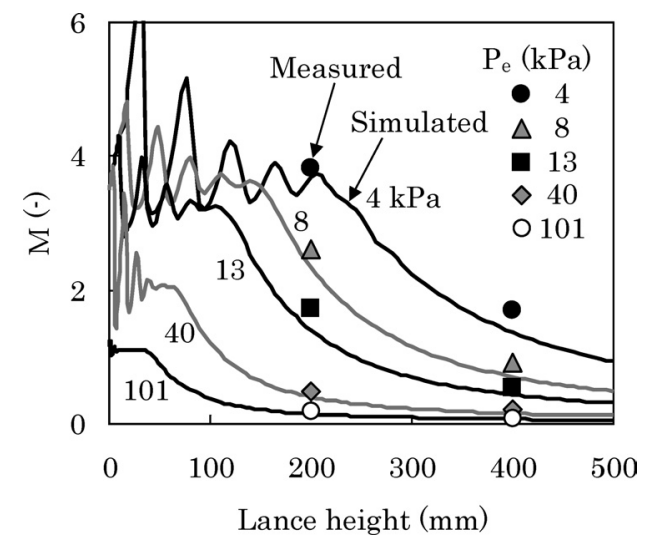

Fig. 3. Mach number distribution in the axial direction.

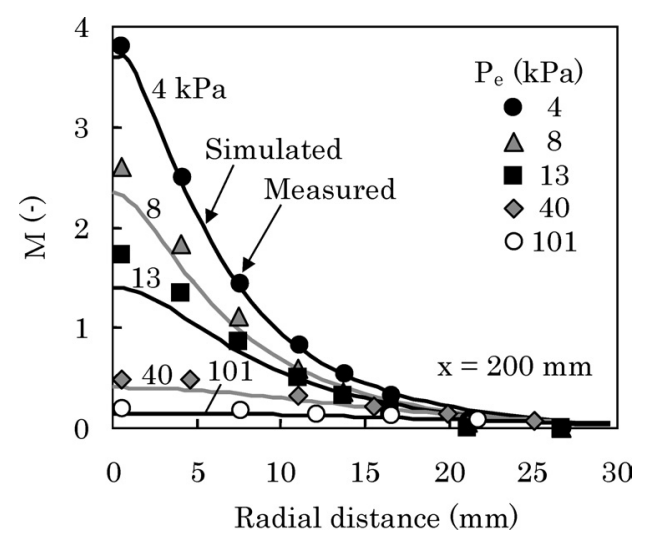

Fig. 4. Mach number distribution in the radial direction.

pressed as the ambient pressure decreases.

Similarly, the distribution of Mach number in the radial direction at $x=200 \mathrm{~mm}$ is shown in Fig. 4 with the Mach number measured in Exp. 1. The simulated results are also in good agreement with the measured data. Using these data, the spreading angle of the jet was examined, as described in the following section.

When the ambient pressure changes, the Mach number at the nozzle exit, $M_{\mathrm{o}}$, which is nearly equivalent to the Mach number at the potential core, also changes as shown in Eq. (1). Then, the Mach number at the nozzle exit was obtained as the simulated Mach number at the point where jet attenuation began and was compared with the theoretical Mach number at the nozzle exit. We can calculate the theoretical Mach number from Eq. (1): in this calculation, $M$ is the Mach number at the nozzle exit and $P$ is the backpressure in the nozzle. The nozzle is designed for an ambient pressure of $8 \mathrm{kPa}$ as previously stated. Therefore, the simulated $M_{\mathrm{o}}$ for $8 \mathrm{kPa}$ had the same value as the theoretical $M_{\mathrm{o}}$; however, the simulated $M_{\mathrm{o}}$ was smaller than the theoretical $M_{\mathrm{o}}$ for other ambient pressures. This is presumably caused by incorrect expansion of the jet at the nozzle exit. The results shown in Figs. 3 and 4 include the effects of such incorrect expansion as will hereinafter be described in detail.

The simulated Mach number in the axial direction was compared with the measured Mach number in Exp. 1 as shown in Fig. 5. The simulated results are in good agreement with the measured data and the numerical simulation method was validated for the supersonic jet simulation under reduced pressure. However, there are slight differ-

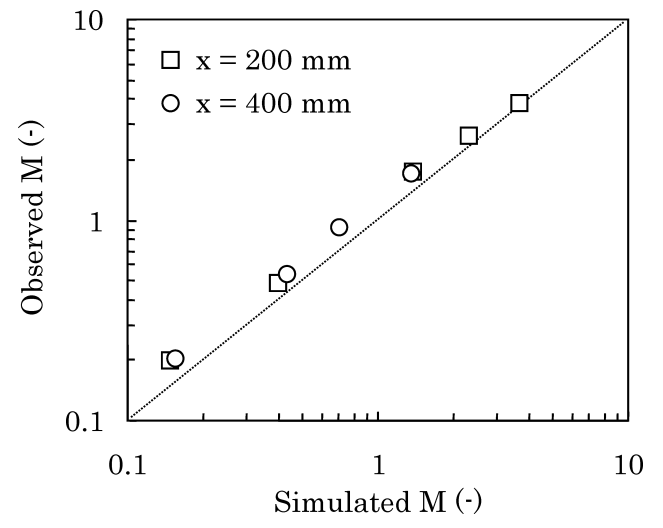

Fig. 5. Comparison between measured and simulated Mach number at nozzle exit.

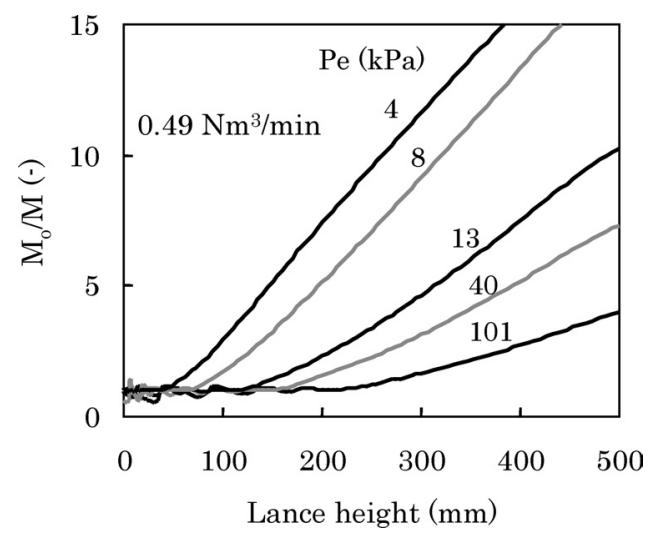

Fig. 6. Distribution of $M_{\mathrm{o}} / M$ in the axial direction.

ences between measured and simulated data, especially in the low $M$ region. Therefore, more optimization is needed for the complete accord in the future.

\subsection{Modeling of Jet in the Axis Direction under Re- duced Pressure}

The results of numerical simulation were validated as noted above. When the nozzle design is fixed, numerical simulation is very useful for understanding the jet behavior. However, when the nozzle shape is not known, numerical simulation is not the best method for optimizing the topblown conditions. We required a huge amount of time just to obtain an outline of the jet behavior. In such a case, a simpler model is needed for examining the nozzle and topblown conditions. Therefore, jet behavior under reduced pressure was modeled with simple equations using the results obtained from the numerical simulation, and the model was used to examine the jet behavior.

$M_{\mathrm{o}} / M$ obtained from the numerical simulation is shown in Fig. 6 as a function of the lance height, $x$. In this study, $M_{\mathrm{o}} / M$ remains equal to unity in the low lance height region where the potential core region is located and increases linearly with the lance height as shown in Fig. 6. Therefore, we adopted the distribution of Mach number in the axial direction as follows:

$$
\frac{M}{M_{\mathrm{o}}}=\frac{K}{(x / D)-\alpha}
$$

where $M_{\mathrm{o}}$ is the Mach number at the nozzle exit obtained 
from the numerical simulation and includes the effects of incorrect expansion. It is identical to the theoretical value obtained by Eq. (1) when a suitable nozzle design is used for correct expansion at the nozzle exit. In addition, the theoretical nozzle exit diameter for each blowing condition, $D,{ }^{11)}$ is used in Eq. (2) to eliminate the geometric influence of the nozzle exit diameter. $K$ and $\alpha$ are parameters and the dimensionless apparent potential core length, $L_{\mathrm{p}} / D$, is equivalent to $K+\alpha$.

Using these equations, parameters were calculated from the results of the numerical simulation. Figure 7 shows the attenuation parameter, $K$, as a function of the pressure ratio, $P_{\mathrm{o}} / P_{\mathrm{e}}$, which is an important factor for the Mach number as shown in Eq. (1), where $P_{\mathrm{o}}$ is the backpressure in the nozzle. This figure shows that attenuation of Mach number of the jet is restrained as the ambient pressure decreases. Similarly, Fig. 8 shows the dimensionless apparent potential core length, $L_{\mathrm{p}} / D=K+\alpha$, as a function of the pressure ratio, $P_{\mathrm{e}} / P_{\mathrm{o}}$. The potential core length is extended as the ambient pressure decreases. From these relationships, the characteristics of the jet under reduced pressure can be determined from the pressure ratio, $P_{\mathrm{e}} / P_{\mathrm{o}}$. We were able to obtain the parameters from these relationships under various blowing conditions.

Meanwhile, $M_{\mathrm{o}}$ is affected by the jet expansion condition at the nozzle exit because of incorrect expansion. Therefore, $M_{\mathrm{o}}$ can be expressed as follows:

$$
M_{\mathrm{o}}=M_{\text {theo }}-M_{\text {loss }}
$$

where $M_{\text {theo }}$ is the theoretical $M_{\mathrm{o}}$ and $M_{\text {loss }}$ is the difference

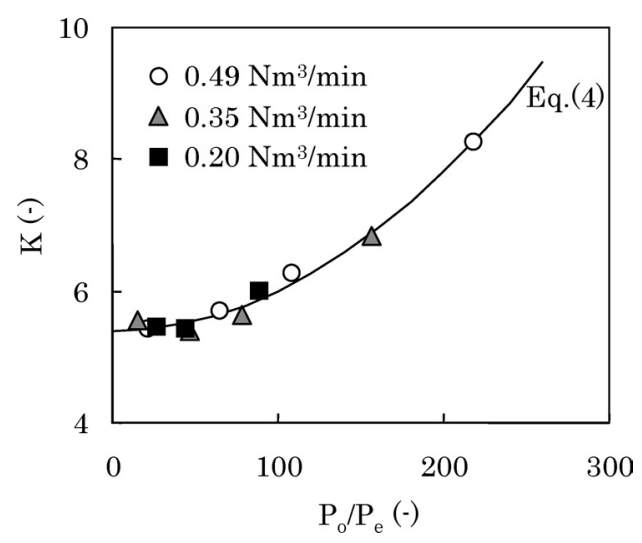

Fig. 7. Effects of pressure ratio on $K$.

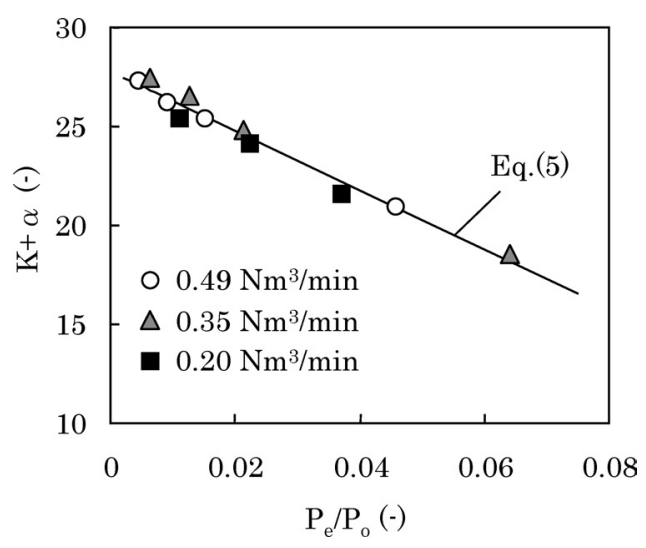

Fig. 8. Effects of pressure ratio on $L_{\mathrm{p}} / D=K+\alpha$. between theoretical $M_{\mathrm{o}}$ and observed $M_{\mathrm{o}}$.

When the ratio of ambient pressure to designed ambient pressure for the nozzle, $P_{\mathrm{e}} / P_{\mathrm{ep}}$, is equal to unity, the jet has the correct expansion and $M_{\text {loss }}$ is theoretically zero. Then, we can obtain $M_{\mathrm{o}}$ as $M_{\text {theo }}$. However, we need $M_{\text {loss }}$ to obtain $M_{\mathrm{o}}$, when $P_{\mathrm{e}} / P_{\mathrm{ep}}$ is not equal to unity. In this study, we obtained $M_{\text {loss }}$ from Eq. (3) with $M_{\text {theo }}$ and the simulated $M_{\mathrm{o}}$. $M_{\text {loss }}$ is shown as a function of $P_{\mathrm{e}} / P_{\text {ep }}$ in Fig. 9.

From the simulated data, approximation formulas were obtained as follows:

$$
\begin{aligned}
& K=6.02 \times 10^{-5} \times\left(P_{\mathrm{o}} / P_{\mathrm{e}}\right)^{2}+5.40 \\
& K+\alpha=-150.4 \times\left(P_{\mathrm{e}} / P_{\mathrm{o}}\right)+27.8 \\
& z=P_{\mathrm{e}} / P_{\mathrm{ep}} \\
& M_{\mathrm{loss}}=2.03(z-1)^{2}, \quad z<1 \\
& M_{\mathrm{loss}}=8.83 \times 10^{-4}(z-1)^{3}-2.44 \times 10^{-2}(z-1)^{2} \\
& +2.34 \times 10^{-1}(z-1), \quad 1<z<7
\end{aligned}
$$

Jet behavior under reduced pressure can be obtained using this simpler jet model based on the numerical simulation.

The jet model under reduced pressure was built from the numerical simulation validated by the data from Exp. 1. Using this model, we examined the effects of the nozzle exit diameter with the data from Exp. 2. Figure 10 shows the relationship between the incorrect expansion parameter, $P_{\mathrm{e}} / P_{\mathrm{ep}}$, and the Mach number at $x=300 \mathrm{~mm}$. In spite of using the same gas flow rate and nozzle throat diameter, the

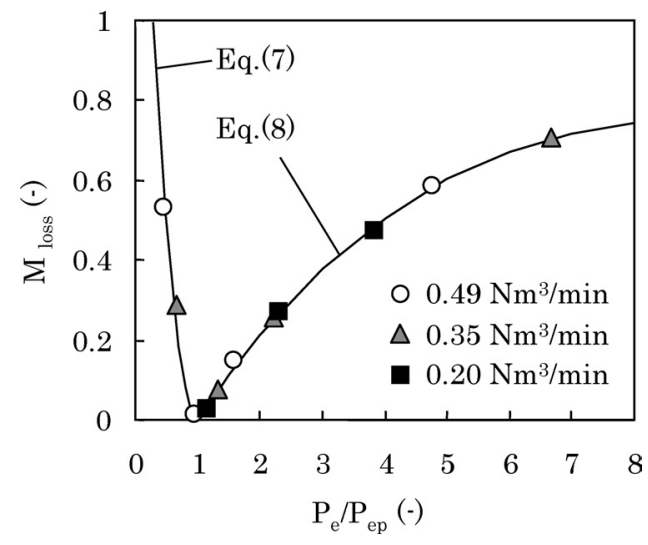

Fig. 9. Effects of incorrect expansion parameter, $P_{\mathrm{e}} / P_{\mathrm{ep}}$, on $M_{\mathrm{loss}}$.

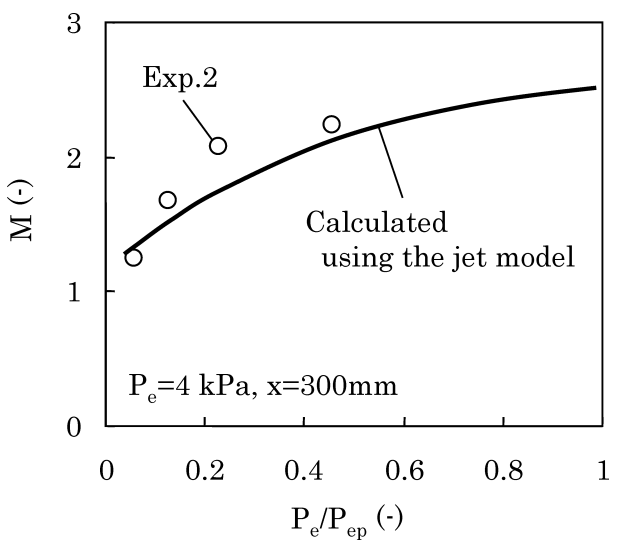

Fig. 10. Effects of nozzle exit diameter on Mach number. 


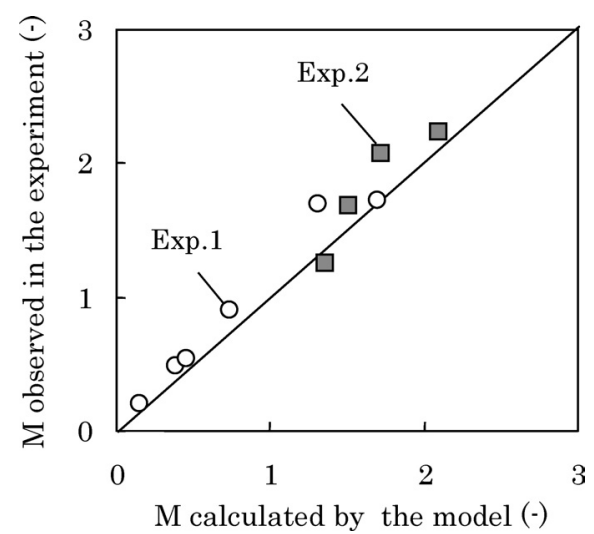

Fig. 11. Validation of model calculation.

measured Mach number decreased with decrease of $P_{\mathrm{e}} / P_{\mathrm{ep}}$. This means that the expansion condition at the nozzle exit affects the jet behavior because of the incorrect expansion. The data obtained in Exp. 2 is in good agreement with the line calculated using the jet model. Therefore, it was clarified that the effects of incorrect expansion at the nozzle exit can be calculated using this jet model.

\subsection{Validation of the Model}

The Mach number calculated using the model was compared with the Mach number observed in Exp. 1 and Exp. 2 concerning the jet attenuation region, $M<3$. In spite of the different experimental conditions, as shown in Table 1, the Mach number calculated using the jet model has a very high correlation with the observed value as shown in Fig. 11. Then, it was validated that the model can estimate the Mach number including the effects of the ambient pressure and the nozzle expansion conditions.

\subsection{Modeling of Jet in the Horizontal Direction under Reduced Pressure}

The jet is also attenuated in the radial direction as shown in Fig. 4. From these data obtained from the numerical simulation, the spreading angle of the jet was examined.

The jet behavior in the radial direction has a Gaussian distribution. ${ }^{13)}$ In this study, it was defined that the jet spread from the center axis to the point of $2 \sigma$, where $\sigma$ is the standard deviation of the Gaussian distribution. Then, the spreading distance from the center axis could be calculated. Spreading behavior in the axial direction is shown in Fig. 12 and the spreading angle can be obtained from this figure.

The defined spreading angle is shown in Fig. 13. The apparent spreading angle is from the nozzle exit, and the actual spreading angle is from the end of the potential core at which jet attenuation starts. For calculating the actual spreading angle, we need the potential core length, which is obtained from Eq. (5) in the jet model. The effects of ambient pressure on the spreading angle are shown in Fig. 14. The apparent spreading angle decreases as the ambient pressure decreases. On the other hand, the actual spreading angle is less affected by the ambient pressure. Therefore, the effects of ambient pressure on the apparent spreading angle are presumably caused by the change in potential core length. In other words, the jet is attenuated with almost the same spreading angle after the potential core region,

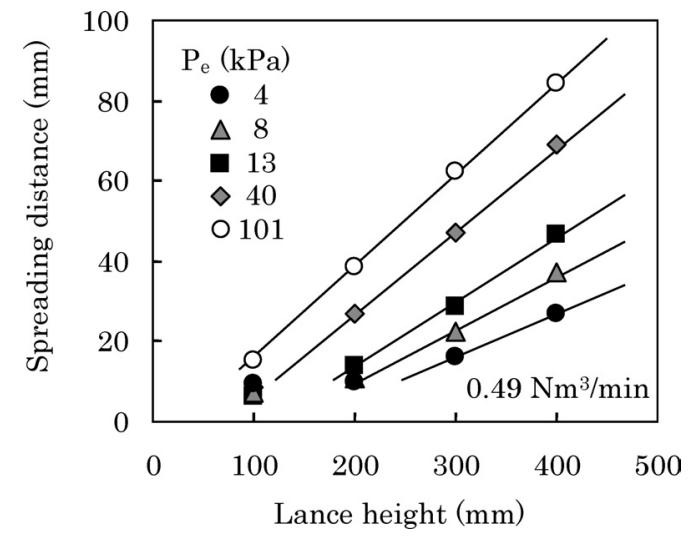

Fig. 12. Spreading behavior in the axial direction.

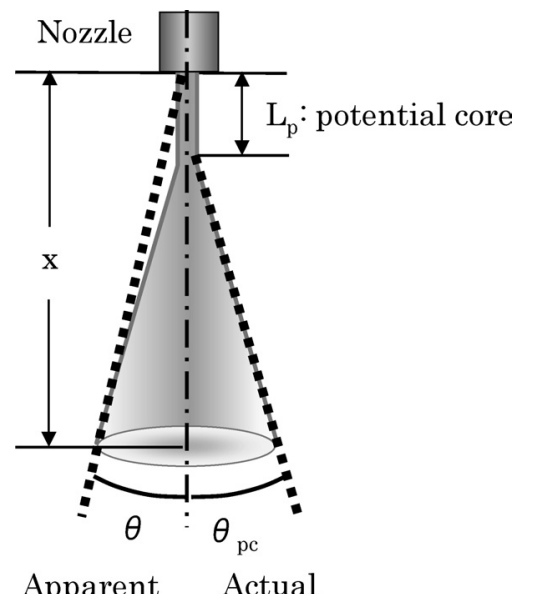

Apparent Actual

Fig. 13. Definition of the spreading angle.

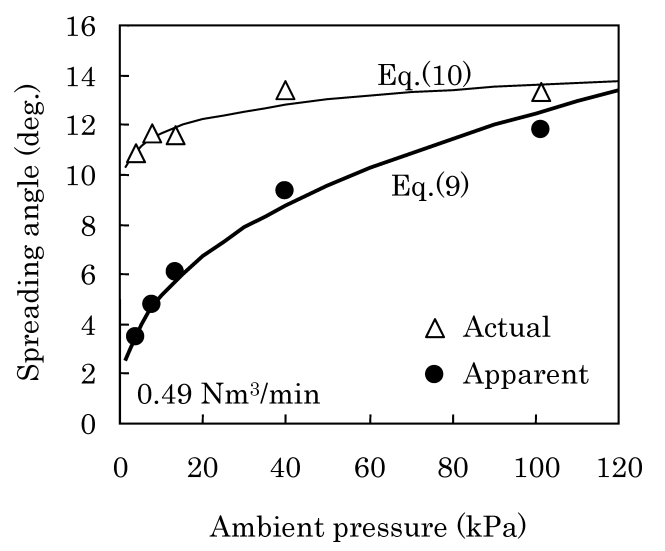

Fig. 14. Effects of ambient pressure on spreading angle.

even if the ambient pressure changes. This can be explained by considering the effects of the ambient density change under the reduced pressure because the spreading angle is less affected by the ambient density under the atmospheric pressure. ${ }^{14)}$

Approximation formulas are obtained from Fig. 14 as follows:

$$
\begin{gathered}
\theta=2.14 \times P_{\mathrm{e}}^{0.383} \\
\theta_{\mathrm{pc}}=10.0 \times P_{\mathrm{e}}^{0.067}
\end{gathered}
$$

where $\theta$ is the apparent spreading angle of the jet and $\theta_{\mathrm{pc}}$ is the actual spreading angle. 


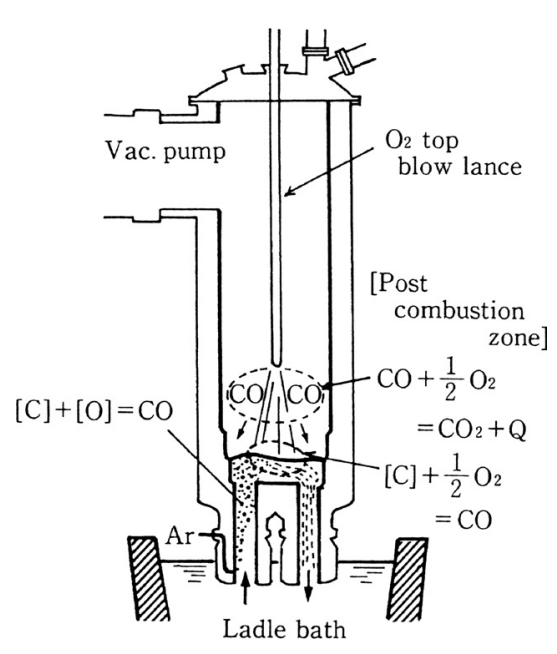

Fig. 15. Schematic illustration of KTB.

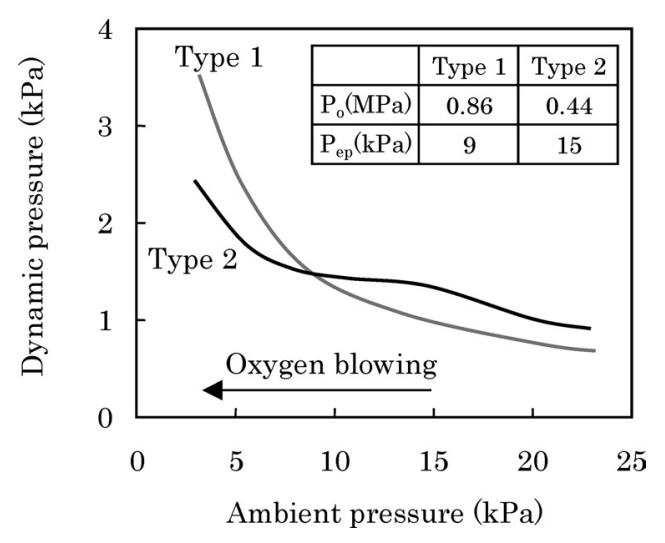

Fig. 16. Dynamic pressure calculated from the jet model.

\section{Application for the RH Process}

An experiment on decarburization of the top-blown oxygen was carried out using the RH-KTB process ${ }^{15)}$ on a 350ton scale. In this experiment, two operations were conducted with different top-blowing Laval nozzles, Type 1 and Type 2. The oxygen blowing rate was similar for both operations; however, the nozzle conditions differed. For Type 1, the backpressure in the nozzle, $P_{\mathrm{o}}$, and the ambient pressure for correct expansion at the nozzle exit, $P_{\mathrm{ep}}$, were $0.86 \mathrm{MPa}$ and $9 \mathrm{kPa}$, respectively. On the other hand, for Type 2, $P_{\mathrm{o}}$ and $P_{\text {ep }}$ were $0.44 \mathrm{MPa}$ and $15 \mathrm{kPa}$, respectively. Initial carbon content was approximately 0.05 mass $\%$ for both operations.

Ambient pressure was reduced from the start of the operation and oxygen was blown when the ambient pressure reached $15 \mathrm{kPa}$; it stopped blowing after a certain period of time. Ambient pressure decreased during the oxygen blowing and it was approximately $3 \mathrm{kPa}$ at the blowing end. Carbon content at the end of the oxygen blowing was approximately 0.02 mass $\%$ for both operations. In this experiment, the dynamic pressure of the top-blown oxygen jet changed during the oxygen blowing because of the ambient pressure change. Dynamic pressure at the metal surface was calculated using the jet model proposed above and shown in Fig. 16. Dynamic pressure typically increased as the ambient pressure decreased in both operations; however, the detailed behavior differed. The dynamic pressure of the Type 1 noz-

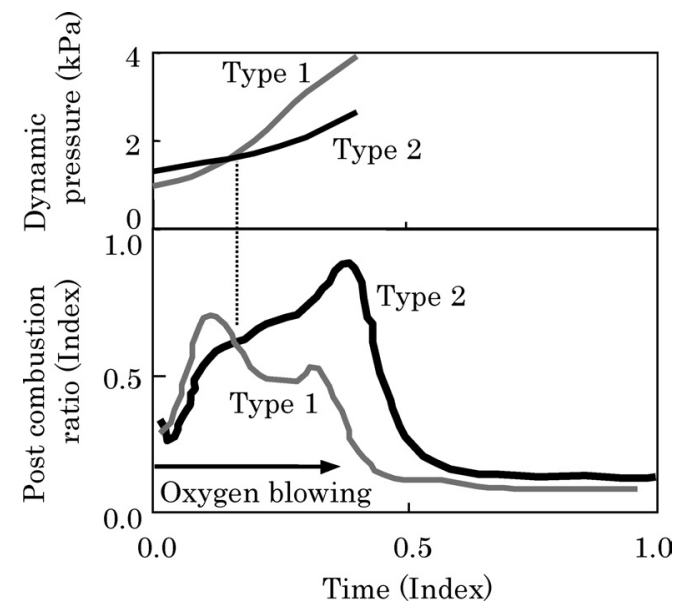

Fig. 17. Post-combustion behavior during RH operation.

zle was lower at the initial stage of blowing than that of the Type 2 nozzle and then increased at the final stage of blowing. On the other hand, the dynamic pressure of the Type 2 nozzle had a relatively mild shift. As shown in Fig. 16, the behavior of the top-blown oxygen under reduced pressure can be quantitatively understood with the jet model.

The post-combustion ratio, PCR, of the experiment is shown in Fig. 17. Dynamic pressure at the metal surface during the oxygen blowing is also shown in Fig. 17 using the calculated results in Fig. 16. The PCR of the Type 1 nozzle was higher at the initial stage of blowing than that of the Type 2 nozzle. This is explained by the lower dynamic pressure calculated from the jet model. At the latter stage of the oxygen blowing, the PCR of the Type 2 nozzle was higher than that of the Type 1 nozzle, presumably because of the milder increase in the dynamic pressure of the Type 2 nozzle.

Further study is needed to define the effects of dynamic pressure on the PCR or decarburization behavior under reduced pressure in the vacuum process. However, using the jet model calculation of the dynamic pressure, we could grasp the real phenomena caused by the difference in nozzle conditions as shown here. In addition, it is considered that the jet model can be useful for optimization of topblown oxygen conditions.

\section{Conclusions}

Supersonic jet behavior under reduced pressure was investigated by measuring the dynamic pressure of the jet in a reduced chamber, and the results were compared with those obtained by numerical simulation. The results showed that the attenuation of Mach number of the jet is restrained and the potential core length is extended under reduced pressure.

Jet behavior was considered using numerical simulation and a simpler model was proposed. This model can be used to estimate the Mach number of the jet with the effect of reduced ambient pressure and the jet expansion condition of the nozzle.

The experiment was carried out using an actual RH-KTB operation, and post-combustion behavior could be explained using the jet model calculation. 


\section{Nomenclature}

$A_{\mathrm{e}}: \quad$ Area of the nozzle exit $\left(\mathrm{m}^{2}\right)$

$A_{\mathrm{t}}$ : Area of the nozzle throat $\left(\mathrm{m}^{2}\right)$

$D$ : Theoretical nozzle exit diameter $(\mathrm{mm})$

$d_{\mathrm{e}}$ : nozzle exit diameter $(\mathrm{mm})$

$d_{\mathrm{t}}$ : nozzle throat diameter $(\mathrm{mm})$

$K$ : Attenuation parameter $(-)$

$L_{\mathrm{p}}$ : Apparent potential core length $(\mathrm{mm})$

$M$ : Mach number (-)

$M_{\mathrm{o}}$ : Mach number at the nozzle exit (-)

$M_{\text {theo }}$ : Theoretical Mach number at the nozzle exit (-)

$M_{\text {loss }}$ : Difference between $M_{\mathrm{o}}$ and $M_{\text {theo }}(-)$

$P$ : $\quad$ Pressure $(\mathrm{kPa})$

$P_{\mathrm{e}}:$ Ambient pressure $(\mathrm{kPa})$

$P_{\mathrm{ep}}$ : Ambient pressure for correct expansion $(\mathrm{kPa})$

$P_{\mathrm{o}}$ : Backpressure in the nozzle $(\mathrm{kPa})$

$x$ : Lance height (mm)

$y$ : Radial distance from center axis of the jet (mm)

$\alpha$ : Core parameter $(-)$

$\gamma:$ Ratio of heat capacity $(-)$

$\theta: \quad$ Apparent spreading angle of the jet (deg.)

$\theta_{\mathrm{pc}}:$ Actual spreading angle of the jet (deg.)

$\sigma$ : Standard deviation of the Gaussian distribution

\section{REFERENCES}

1) R. Imai, K. Kawakami, S. Miyoshi and S. Jinbo: Nippon Kokan Tech. Rep.-Overseas, 8 (1968), 9.

2) S. Ito and I. Muchi: Tetsu-to-Hagané, 55 (1969), 1152.

3) M. Shimada, M. Ishibashi and H. Morise: Seitetsu Kenkyu, 266 (1969), 8855.

4) K. Naito, Y. Ogawa, T. Inomoto, S. Kitamura and M. Yano: ISIJ Int., 40 (2000), 23.

5) I. Sumi, R. Kawabata, Y. Kikuchi, H. Igarashi, H. Nakabayashi and N. Kobayashi: CAMP-ISIJ, 14 (2001), 876.

6) I. Sumi, R. Kawabata, T. Ishii and Y. Kikuchi: CAMP-ISIJ, 13 (2000), 970.

7) I. Sumi, R. Kawabata, Y. Kikuchi, S. Kohira and T. Isawa: Steel Res., 74 (2003), 14.

8) Y. Tago and Y. Higuchi: ISIJ Int., 43 (2003), 209.

9) Y. Kotani, T. Ikeda and K. Ishihara: Tetsu-to-Hagané, 62 (1976), 1795.

10) K. Naito, K. Miyamoto and S. Kitamura: CAMP-ISIJ, 18 (2005), 164.

11) D. R. Poirier and G. H. Geiger: Transport Phenomena in Materials Processing, TMS, Pennsylvania, (1994), 158.

12) J. Kondo: High Speed Aerodynamics, Corona, Tokyo, (1977), 82.

13) H. Ishigaki: Trans. Jpn. Soc. Mech. Eng. B, 48-433 (1982), 1692.

14) H. Ishigaki: Trans. Jpn. Soc. Mech. Eng. B, 48-433 (1982), 1701.

15) K. Kameyama, H. Nishikawa, M. Aratani, R. Asaho, N. Tamura and K. Yamaguchi: Kawasaki Steel Giho, 23 (1991), 136. 